\title{
Percepciones y expectativas de docentes de ingeniería y educación en torno a la retroalimentación en tareas de escritura
}

\author{
Débora D. Cuevas-Solar(1) y Beatriz M. Arancibia ${ }^{(1.2)}$ \\ (1) Universidad Católica de la Santísima Concepción, Facultad de Educación, Dpto. de Ciencias de Lenguaje y Literatura, \\ Alonso de Ribera 2850, Concepción - Chile (e-mail: daniela.cuevas@ucsc.cl) \\ (2) Universidad Católica de la Santísima Concepción, Facultad de Educación, Dpto. de Ciencias de Lenguaje y Literatura, \\ Investigadora CIEDE - UCSC, Alonso de Ribera 2850, Concepción - Chile (e-mail: barancib@ucsc.cl)
}

Recibido Oct. 11, 2019; Aceptado Dic. 6, 2019; Versión final Feb. 7, 2020, Publicado Ago. 2020

\begin{abstract}
Resumen
El objetivo de este estudio es analizar las percepciones y expectativas de un grupo de docentes de Ingeniería y Educación en torno a la retroalimentación a la escritura de sus estudiantes. Participaron voluntariamente seis docentes por área disciplinar. Se les realizó una entrevista en profundidad semiestructurada que fue analizada con NVivo v.11. Las expectativas de los docentes de Ingeniería respecto de las tareas de escritura de sus estudiantes es que demuestren el conocimiento que poseen o que adquirieron con la realización de la tarea. Los participantes de Educación esperan que las tareas de escritura den cuenta de una buena ortografía, redacción, coherencia y un buen contenido en los textos. Sobre la recepción de la tarea y de la retroalimentación por parte de los estudiantes, se concluye que los docentes entrevistados perciben una mala recepción de las tareas de escritura, pero una buena acogida hacia la retroalimentación que se les proporciona.
\end{abstract}

Palabras clave: retroalimentación; escritura en las disciplinas; visiones docentes

\section{Perceptions and expectations of education and engineering professors about feedback on written assignments}

\begin{abstract}
The purpose of this study is to analyze the perceptions and expectations of a group of engineering and education professors regarding the feedback from student written assignments. Six professors from each faculty, education and engineering, volunteered for this study. Each professor answered an in-depth semistructured interview that was analyzed using NVivo v.11. The results show that the expectations of the engineering teachers regarding their students' written assignments is for students to show the knowledge level they already had or that they show the knowledge acquired while doing a writing task. Education teachers expect students to show good spelling, penmanship, coherence, and good content in their writings. In regards to the reception and feedback from students, it is concluded that the professors perceived a negative reception from their students to written assignments, but the professors also perceived a positive reaction from their students to the feedback provided.
\end{abstract}

Keywords: feedback; discipline writing; teaching views 


\section{INTRODUCCIÓN}

Actualmente, los modelos educativos de las universidades tienden a basarse en un currículum orientado al logro de competencias evidenciables en resultados de aprendizaje de distinta naturaleza, con pertinencia al perfil de egreso institucional y al de las carreras en particular. Entre los criterios que fijan las instituciones de educación superior para la implementación de sus modelos educativos, se establece el uso de metodologías activas de enseñanza y de instancias de monitoreo y seguimiento de los aprendizajes que los estudiantes deben adquirir para demostrar las competencias declaradas en los perfiles de egreso. De acuerdo con esto, las prácticas evaluativas se tienen que concebir e implementar como instancias de formación, para lo cual es fundamental proveer información oportuna a los estudiantes sobre sus avances, logros y dificultades.

En el contexto de lo ya señalado, la retroalimentación que los docentes proveen durante el proceso de enseñanza-aprendizaje juega un rol fundamental. Desde la perspectiva de la evaluación formativa entendida como parte del proceso de enseñanza - aprendizaje, se requiere de una retroalimentación continua y oportuna (Monedero, 1998). De esta forma, los docentes pueden ofrecer a sus estudiantes oportunidades para ser aprendices críticos y proactivos. En este sentido, como sostienen Boud y Molloy (2015, p.13), "la retroalimentación es el mecanismo a través del cual los alumnos descubren si están siendo exitosos en sus tareas y si están listos para cumplir con las expectativas". Por tanto, si queremos que los estudiantes logren alcanzar los objetivos que se les ha propuesto para el aprendizaje o la evaluación, se les debe proveer la información que les permita saber qué hicieron, cómo lo hicieron y qué les falta para lograr el aprendizaje deseado. En términos de Vigotsky (1978/1997), la retroalimentación les indica cuál es su estado actual como aprendices y qué tan cerca están de alcanzar lo que se espera de ellos. Para esto es necesaria la interacción colaborativa entre los estudiantes, docentes e institución. Los esfuerzos del aprendiz son importantes, pero también lo son las condiciones y oportunidades que los docentes ofrecen para que los estudiantes pongan en marcha su actividad cognitiva (Carlino, 2005). Respecto a esto, Boud y Molloy (2015) postulan que "una de las razones clave para centrarnos en la retroalimentación es que en general se asume como una parte importante del aprendizaje y se refiere a una parte igualmente significativa de la vida del aprendiz" (p.13).

En los últimos años, diversos autores se han interesado por investigar en torno a la retroalimentación a la escritura académica de los estudiantes. Las investigaciones asumen que la escritura en la universidad es un proceso complejo que requiere andamiaje, por lo que la retroalimentación es fundamental. La atención de los especialistas se ha centrado principalmente en estudiar los tipos de comentarios que proveen los docentes a las tareas de escritura (Arancibia et al., 2019), la eficacia de los comentarios (Hartshorn y Evans, 2015), la retroalimentación entre pares (Huisman et al., 2018), entre otros. No obstante, son escasos los estudios sobre la visión y pensamiento de los propios docentes universitarios sobre la retroalimentación que proporcionan a la escritura de sus estudiantes. Por este motivo, esta investigación indaga en esta temática que ha sido poco explorada. Específicamente, se busca analizar las expectativas y percepciones de profesores de Ingeniería y de Educación de una Universidad del Consejo de Rectores respecto a la retroalimentación que otorgan a las tareas de escritura y respecto al desempeño y (re)acciones de sus estudiantes como destinatarios de dicha retroalimentación.

El supuesto en que se basa la investigación es que conocer las percepciones y expectativas de los profesores nos abre una ventana a su dispositivo epistemológico interno, el cual puede operar como un filtro de su actuar pedagógico, gatillando acciones que favorecen o bien dificultan el progreso de los estudiantes como escritores noveles en su disciplina. Aunque la comunicación escrita es una competencia transversal en la formación universitaria, las investigaciones muestran diferencias en los usos y funciones que se le asigna al interior de las distintas comunidades discursivas (v.g. Hyland, 2012, 2015; Marinkovich et al., 2018). Por lo tanto, interesa averiguar si tales diferencias se observan también en las percepciones y expectativas que los profesores manifiestan según sean docentes de Ingeniería o de Educación. En este sentido, el presente estudio proporciona información que puede resultar muy valiosa para orientar el uso de la retroalimentación como un recurso de aprendizaje en la enseñanza de la escritura académica en las disciplinas seleccionadas.

\section{OTROS ANTECEDENTES}

En este apartado se encuentran algunos fundamentos teóricos que respaldan esta investigación. Los conceptos que se desarrollan teóricamente son: escritura académica, expectativas, percepciones, retroalimentación en la evaluación formativa y retroalimentación en la escritura.

\section{Escritura académica}

Con el ingreso a la universidad los estudiantes se enfrentan a nuevas experiencias de lectura y escritura que corresponden a prácticas de alfabetización académica necesarias para acceder a la cultura universitaria y formarse como futuro profesional en un campo disciplinar determinado. Como sostiene Castelló et al. (2012), 
tales prácticas requieren competencias no necesariamente transferibles desde experiencias de aprendizaje anteriores. Es más, el paso de la educación secundaria a la universitaria no es un proceso gradual de cambio y adaptación, sino más bien "un impacto comunicativo, cultural, cognitivo, académico y social" (Navarro et al., 2019, p. 77). Uno de los mayores desafíos que este impacto representa para los estudiantes, es aprender a comunicar por escrito propósitos y contenidos propios de su área de formación profesional-disciplinar a través de géneros discursivos especializados. Diversos autores coinciden en plantear que esta es una tarea compleja, pues normalmente se trata de géneros que los estudiantes no tienen incorporados en su repertorio al momento de ingresar a la universidad, no obstante, constituyen un medio indispensable en la dinámica de transmisión-construcción de los saberes disciplinares dentro de un campo de estudio (Carlino, 2008; Camps y Castelló, 2013; Marinkovich y Córdoba, 2014; Navarro, 2018). No sólo eso, forman parte de las prácticas colectivas de la comunidad disciplinar, por lo que su manejo es fundamental en el desarrollo del sentido de identidad y pertenencia de los estudiantes (Hyland, 2015).

De acuerdo con lo ya señalado, el concepto de alfabetización académica nos parece fundamental, pues permite entender el proceso de enseñanza y aprendizaje de la escritura académica en la universidad como parte de un proceso de enculturización disciplinar (Carlino, 2013; véase Navarro, 2018). Al respecto, es importante relevar que "la alfabetización académica no es una propuesta para remediar la mala formación de quienes llegan a la universidad" (Carlino, 2005, p.7). Más bien, según Marinkovich y Córdoba (2014), la alfabetización académica es la mediadora entre la relación sujeto/objeto, lo que conlleva a enfatizar en su función epistémica. Sin duda, esto también supone la construcción de la propia identidad como escritor/autor $y$, por lo tanto, la enseñanza de la escritura académica en la universidad tiene que propiciar que los estudiantes sean conscientes de sus propósitos discursivos y aprendan a usar el discurso académico en función de sus objetivos (Camps y Castelló, 2013).

En este contexto, es necesario enseñar explícitamente a los estudiantes cómo responder a las tareas de escritura que deben llevar a cabo. Côrte (2018) plantea que los programas de escritura deben formular una propuesta de actividades de diferentes tipos de textos y con pautas definidas, incluyendo los tres componentes básicos, a saber, conocimiento, prácticas y actitudes. Así pues, los docentes que dan las tareas de escritura deben proporcionar descripciones claras respecto a sus características y propósitos, y evaluarlas conforme a esos criterios. De igual forma, los textos tienen que variar en su tipo y complejidad para que los estudiantes tengan la oportunidad de desarrollar sus habilidades de escritura adecuando su actuación como escritores a las características de la tarea y al contexto en el cual la realizan. De esta forma, la actividad discursiva de los alumnos se va encauzando, en las diferentes áreas disciplinares, hacia la producción de géneros discursivos específicos al servicio de la producción/construcción/comunicación de conocimiento, de acuerdo a las convenciones propias de su área de formación, como propone el enfoque conocido como escritura en las disciplinas o por la sigla en inglés WID (Writing in the disciplines, Bazerman et al., 2016).

\section{Expectativas}

Las expectativas son entendidas como aquello razonable que puede ocurrir y que se formula mediante la anticipación (Valle y Núñez, 1989). Por ello, en relación al ámbito educativo, Mares, Martínez y Rojo (2009) postulan que "una expectativa no fundamentada se confirma sencillamente porque se espera su ocurrencia; en el aula esto significa que las opiniones del maestro sobre sus estudiantes ocasionan las conductas esperadas; por lo tanto, a pesar de mostrar cambios, las expectativas de su profesor se sostendrán" (p. 974). Respecto a esto, Finn (1972) citado por Mares et al., (2009, p. 971) postula que "las expectativas de los docentes sobre sus alumnos, producto del concepto que tienen de ellos, pueden convertirse en un cumplimiento del pronóstico de rendimiento académico y disciplina escolar". Es decir, la imagen o concepto del docente respecto al estudiante puede afectar - e incluso determinar - el desempeño de este, pues lo que se espera del estudiante se relaciona con las exigencias que el profesor establece para la tarea y con el tipo de retroalimentación que proporciona. En cuanto a las investigaciones, las expectativas han sido abordadas en relación a temas como: Expectativas laborales de los egresados de Comunicación (Pérez Serrano et al., 2015); Expectativas y cambios metodológicos: una visión desde el mundo de los profesores (Schiefelbein y Schiefelbein, 2017), entre otros. Cada uno de estos estudios apunta a que las expectativas sobre un hecho, suceso o persona están mediadas por el concepto o concepción que se tiene que ello.

\section{Percepciones}

Las percepciones influyen en nuestras actitudes y estas a su vez afectan nuestro comportamiento. Vargas (1994) plantea que "en el proceso de la percepción se ponen en juego referentes ideológicos y culturales que reproducen y explican la realidad y que son aplicados a las distintas experiencias cotidianas para ordenarlas y transformarlas" (p. 49). Entonces, las percepciones orientan y condicionan las acciones o conductas de las personas. Por lo tanto, las percepciones que los profesores tienen sobre la retroalimentación también pueden incidir en sus prácticas (Contreras-Pérez y Zúñiga-González, 2017). 
Vargas (1994) especifica que las percepciones han sido abordadas desde la psicología como un proceso cognitivo que consiste en el reconocimiento, interpretación y significación para elaborar un juicio en relación al estímulo que se obtiene del entorno. Sin embargo, las percepciones no son sólo eso, debido a que dependen de la ordenación, clasificación y elaboración de categorías que se comparan con los estímulos que el sujeto recibe. De esta forma, se configuran los referentes perceptuales a través de los cuales se identifican las nuevas experiencias sensoriales, transformándolas en eventos reconocibles y comprensibles (Vargas, 1994). Es decir, las percepciones son los referentes conceptuales que las personas poseen en relación a la experiencia y que van adquiriendo significado en la medida en que son interpretadas e identificadas por los sujetos.

Desde la investigación, las percepciones han sido abordadas en estudios como: Percepciones de alumnos, egresados y profesores sobre los sistemas de evaluación del aprendizaje (Galán et al., 2015); percepciones acerca de la integración de las TIC en el proceso de enseñanza-aprendizaje de la universidad (Morales et al., 2015); percepciones sobre la integración de modelos pedagógicos en la formación del profesorado: la simulación y juego, y el flipped classroom (Angelini y García-Carbonell, 2015); percepciones de directivos y profesorado de educación física sobre las competencias básicas (Alcalá et al., 2015), Enseñar a escribir un artículo de investigación mediante la revisión colaborativa llamada Percepciones de los estudiantes (Corcelles et al., 2017).

\section{Retroalimentación en la evaluación formativa}

Según Rosales (2014), "la evaluación constituye una reflexión crítica sobre todos los momentos y factores que intervienen en el proceso didáctico a fin de determinar cuáles pueden ser, están siendo o han sido, los resultados del mismo" (p.15). Desde esta perspectiva, la evaluación conlleva entregar información desde el que evalúa al que es evaluado. Específicamente, la evaluación formativa, según Brown y Pickford (2014), "es principalmente un medio por el que los profesores pueden apoyar a sus alumnos y estimular su progreso, facilitándoles una retroinformación que tenga sentido para el individuo" (p. 24). De esta forma, la retroalimentación es una de las herramientas instruccionales más poderosas, pues permitiría cerrar la brecha entre el desempeño actual del estudiante y el esperado durante el proceso de aprendizaje, lo que Vygotsky (1978 - 1997) denomina "Zona de desarrollo próximo".

Contreras-Pérez y Zúñiga-Gonzales (2017) plantean que la retroalimentación es considerada parte de la evaluación formativa debido a que es un proceso dialógico que inicia el profesor con el propósito de desarrollar en los estudiantes habilidades de autoevaluación y monitoreo. El medio para ello son los comentarios y sugerencias que le ofrece al estudiante en relación a sus resultados de aprendizaje o a lo observado en la ejecución de alguna evaluación o actividad. Las actuales concepciones evaluativas consideran que estas prácticas de retroalimentación involucran a los docentes y estudiantes en un análisis y diálogo de aprendizaje y son "una actividad clave en el proceso de enseñanza-aprendizaje del alumno, ya que implica darle información que le ayude a cumplir con los objetivos de aprendizaje" (Martínez y Vargas, 2014, p. 200). Canabal y Margalef (2017), por su parte, plantean que la retroalimentación es un apoyo fundamental para el aprendizaje, no solo desde la perspectiva de la "retroalimentación", sino también como "feedforward". Como también explican Tapia-Ladino et al. (2016), siguiendo a Hounsell (2008), el proceso de retroalimentación corresponde a la entrega de información de un agente a otro para que este mejore su desempeño actual en una tarea dada y pueda transferir dicha información en tareas futuras, haciendo uso de las habilidades o estrategias adquiridas con la ayuda de procesos de retroalimentación anteriores.

Una adecuada retroalimentación e instrucciones precisas por parte del docente son cruciales para la buena ejecución de una tarea y la consecución de un objetivo fijado anteriormente. Según Irigoyen et al. (2002) las instrucciones y la retroalimentación funcionan como referencia de lo que tiene que hacer el sujeto acerca del resultado de su respuesta ante un criterio relacional. Desde el punto de vista de este autor, instrucción y retroalimentación se encuentran en el mismo nivel. Sin instrucción y sin retroalimentación la tarea puede doblar su nivel de dificultad, por lo que el estudiante no puede ajustar su desempeño en relación a la tarea o meta a alcanzar. Tanto la instrucción como la retroalimentación buscan eliminar la suposición y dar a conocer el propósito lo más claro posible, de modo que no haya dificultades al momento de llevar a cabo lo solicitado. No obstante, para que esta retroalimentación sea efectiva se debe considerar que el aprendiz debe poseer una meta de aprendizaje, para que así se compare el nivel actual del desempeño con dicha meta y se comprometa en una acción que conduzca a algún cierre de la brecha (Chappuis et al., 2012).

Con base en lo expuesto con anterioridad, es posible afirmar que la retroalimentación que ofrecen los docentes a sus estudiantes debe permitir detectar errores y proporcionar, a la vez, distintas vías para la consecución del objetivo propuesto. Para ello es importante que sea inmediato, específico y que facilite la acción (Chan et al., 2014). Siempre la retroalimentación debe apuntar a mejorar el desempeño de los 
estudiantes, no sólo ser un comentario positivo o negativo, sino ser a la vez un medio que guíe al aprendiz hacia una mejora constante de sus habilidades y capacidades. Por ello, la retroalimentación contribuiría a la adquisición de una competencia, dado que el logro de ella tiene considerado trayectos de aprendizajes. No obstante, para que la evaluación esté orientada al aprendizaje, se debe incluir información que pueda ayudar al estudiante a que en el futuro tenga un mejor desempeño cuando haga tareas similares. En este sentido, para que la retroalimentación también sea feedforward de calidad, no solo tiene que informar de los errores, sino también proveer información suficiente y oportuna - ya sea por escrito (en digital o de forma manual) o en soportes orales reproducibles- para favorecer el cambio a lo largo de un proceso de aprendizaje, destacando los logros del aprendiz y lo que debe repensar o rehacer. Por lo tanto, es recomendable que los comentarios del profesor incluyan explicaciones y proporcionen pistas o sugerencias para mejorar 0 solucionar el problema.

\section{Retroalimentación en la escritura}

La escritura es parte integral de la enseñanza de una disciplina y para ello es necesario establecer instancias de diálogo que faciliten que el estudiante adquiera recursos productivos para desarrollar progresar como escritor En ese contexto, el empleo de adecuadas y oportunas estrategias de retroalimentación del profesorado puede ayudar al estudiante a mejorar de forma constante sus habilidades de escritura (Alvira, 2016). Al respecto, Padilla y López (2019) postulan que el "fuerte apoyo de prácticas de retroalimentación oral y escrita, permite a los estudiantes no solo aprender a escribir académicamente en un proceso recursivo -con numerosos borradores, sino también inscribirse como productores principiantes del conocimiento disciplinar" (p.333). Desde esta perspectiva, indicaciones claras y retroalimentación adecuada permiten que el estudiante aprenda acerca de la disciplina en la cual está inmerso, dado que el docente, quien posee la noción del género, irá otorgando pistas o proporcionando comentarios que acerquen al estudiante al texto meta. En un estudio experimental llevado a cabo por Ortiz (2010), se mostró que cuando los sujetos reciben instrucciones específicas y retroalimentación continua, obtienen un mejor desempeño en la tarea de escritura.

Los estudios sobre retroalimentación a la escritura se han desarrollado fuertemente en los últimos años, especialmente en el ámbito de la enseñanza de inglés como segunda lengua o L2 (Yallop y Leijen, 2018). En ese contexto, el énfasis ha sido la retroalimentación orientada a la corrección de errores lingüísticos (v.g. Ferris, 2010; Ellis, 2009). Sin embargo, en el último tiempo ha ido creciendo el interés por analizar los comentarios que los profesores dirigen a otros aspectos de la escritura, como lo es la aproximación del texto del estudiante al género discursivo que debe producir, especialmente la tesis (Tapia-Ladino et al., 2017; Arancibia et al., 2019). Ochoa-Sierra y Moreno-Mosquera (2019) plantean niveles de caracterización general de los comentarios que se proporcionan a tareas de escritura. Estos niveles son: el nivel pragmático en donde se identifica al emisor y el papel que asume, cómo se percibe al receptor y cuál es la finalidad comunicativa del comentario y el tono con el cual se realiza; el nivel semántico que hace referencia a los aspectos en los que se centra el comentario, es decir, el contenido y, el último, el nivel sintáctico-textual en el cual se da cuenta sobre la cohesión y longitud del comentario.

Respecto a los efectos de la retroalimentación en la escritura de los estudiantes, Chistiansen y Bloch (2016) plantean que los comentarios de los profesores pueden ser muy variados en sus propósitos, al igual que las respuestas de parte de los estudiantes. Esto implica que la efectividad de la retroalimentación sobre la calidad de la escritura depende de diversos factores. Entre ellos, los autores destacan: que la retroalimentación sea provista en ambientes de aprendizaje dialógicos (Dowden et al., 2013); que el profesor tenga clara la meta y comparta los objetivos con sus estudiantes, que los comentarios aludan al desempeño del destinatario y promuevan que este construya su propio aprendizaje (Chappuis et al., 2012); que los comentarios generen oportunidades de mejora y que los estudiantes desplieguen estrategias metacognitivas y de autorregulación que les permitan aprovechar las instancias de retroalimentación para mejorar su escritura (Duijnhouwer, 2010).

\section{METODOLOGÍA}

La presente investigación estuvo orientada por el método cualitativo, bajo el enfoque fenomenológico, el cual según Bautista (2011) "describe las estructuras de la experiencia tal y como se presentan en la conciencia, sin recurrir a teoría, deducción o suposiciones procedentes de otras disciplinas tales como las ciencias naturales" (p. 52). Es decir, se pretende describir y dar a conocer la experiencia tal como se manifiesta y ocurre en la conciencia. Además, "es apropiada para entender y describir aquellos fenómenos humanos que no son explicados desde las teorías positivistas" (Bautista, 2011, p. 53), con el propósito de abordar la realidad del estudio desde un paradigma naturalista, tratando de entenderla dentro del marco de referencia de sus protagonistas. 


\section{Participantes}

La investigación se llevó a cabo en una universidad perteneciente al Consejo de Rectores de la Región del Biobío. Participaron seis docentes de la Facultad de Ingeniería y seis docentes de la Facultad de Educación. El método de selección de los participantes fue no probabilístico, pues estos debían cumplir con los requisitos de dar tareas de escritura y ofrecer retroalimentación por escrito a dichas tareas. Para averiguarlo, se aplicó una encuesta on line y se consultó personalmente. La participación también dependió de la disponibilidad de los docentes, ya que esta debía ser voluntaria. La Tabla 1 presenta las características de los participantes.

Tabla 1. Descripción de los participantes, con información proporcionada por cada uno de los docentes entrevistados.

\begin{tabular}{|l|c|c|c|c|}
\hline & \multicolumn{2}{|c|}{ Género } & \multicolumn{2}{c|}{ Grado } \\
\hline Facultad & Mujeres & Hombres & Magister & Doctorado \\
\hline Ingeniería & 1 & 5 & 2 & 4 \\
\hline Educación & 3 & 3 & 1 & 5 \\
\hline Totales & 4 & 8 & 3 & 9 \\
\hline
\end{tabular}

Para resguardar las consideraciones éticas de la investigación, a todos los docentes se les solicitó la firma del consentimiento informado, el que incluía la autorización para la grabación de la entrevista. En dicho consentimiento se establece que la información proporcionada sería manejada para fines de la investigación y que la identidad de los participantes sería anonimizada.

\section{Técnicas para la recopilación de información}

Para la obtención de la información se realizó una entrevista semiestructurada, la que fue validada mediante el juicio de tres académicos expertos. Uno de ellos es especialista en evaluación, otro en educación universitaria y otro en escritura académica, quienes revisaron cada pregunta considerando su pertinencia en relación a los objetivos que guiaron esta investigación. Como resultado de la consulta a expertos, se obtuvo el instrumento definitivo, el que quedó conformado por nueve preguntas (Tabla 2), organizadas en torno a dos grandes categorías. No se preestablecieron subcategorías con el fin de conocer, naturalmente, las percepciones y expectativas de los docentes.

Tabla 2. Categorías y preguntas derivadas de cada una de ellas

\begin{tabular}{|l|l|}
\hline \multicolumn{1}{|c|}{ Categorías } & \multicolumn{1}{c|}{ Preguntas } \\
\hline Expectativas & $\begin{array}{l}\text { ¿Qué espera usted de las tareas de escritura de sus estudiantes? } \\
\text { ¿Qué espera que los estudiantes hagan con la retroalimentación que le proporciona? } \\
\text { En relación a la retroalimentación que proporciona ¿cómo espera que esto ayude a que sus } \\
\text { estudiantes mejoren en las tareas de escritura? }\end{array}$ \\
\hline Percepciones & $\begin{array}{l}\text { ¿Cómo perciben las tareas de escritura sus estudiantes? } \\
\text { ¿Cómo perciben los estudiantes la retroalimentación que le proporciona a sus informes escritos? } \\
\text { ¿Qué percibe usted que hacen los estudiantes con los comentarios que realiza a los trabajos } \\
\text { escritos? } \\
\text { ¿Cómo percibe usted que la retroalimentación ayuda al estudiante a mejorar sus informes? } \\
\text { ¿Cómo percibe usted que sus prácticas de retroalimentación son efectivas en la realización de } \\
\text { la tarea que lleva a cabo el estudiante? } \\
\text { ¿Percibe usted que los estudiantes mejoran sus tareas de escritura con la retroalimentación que } \\
\text { les proporciona? }\end{array}$ \\
\hline
\end{tabular}

\section{Procedimiento para la obtención de entrevistas}

Para la obtención de las entrevistas se estableció contacto con los participantes mediante correo electrónico en el que se acordó el día y la hora para la realización de la entrevista. Todas fueron realizadas en las respectivas oficinas de los profesores y grabadas con la aplicación Grabadora de voz con un celular Moto G4 plus. Posteriormente fueron transcritas en formato digital (Word) con un sistema de notación simple, respetando en todo su contenido por parte de la investigadora responsable y una ayudante. Finalmente, se llevó a cabo una revisión de ambas transcripciones y se volvieron a escuchar aquellas grabaciones en las cuales existían dudas en algunos segmentos. 


\section{Análisis de la información}

Se efectuó un análisis de contenido de las entrevistas mediante el programa Nvivo v.11. Una vez cargadas las entrevistas, se identificaron y codificaron segmentos de texto en función de los nodos previamente establecidos. De manera recursiva, se fueron desprendiendo del análisis los subnodos o subcategorías, logrando así una representación arbórea y, por lo tanto, jerárquica, de la organización de la información para poder responder a las preguntas de investigación. Durante el proceso se recurrió a la consulta de expertos cuando se estimó necesario en la toma de decisiones respecto a los subnodos y subcategorías que se desprendieron del análisis.

\section{RESULTADOS}

Se presentan los resultados de la investigación en relación a las categorías de expectativas y percepciones organizadas por área disciplinar: profesores Facultad de Ingeniería y profesores Facultad de Educación

\section{Percepciones y expectativas profesores Facultad de Ingeniería}

En la Figura 1 se representa gráficamente las expectativas que poseen los docentes de Ingeniería respecto a las tareas de escrituras de los estudiantes y a la retroalimentación que se les proporciona. Además, con el análisis de Nvivo fue posible desprender de las subcategorías dos subnodos correspondientes a escritura y retroalimentación.

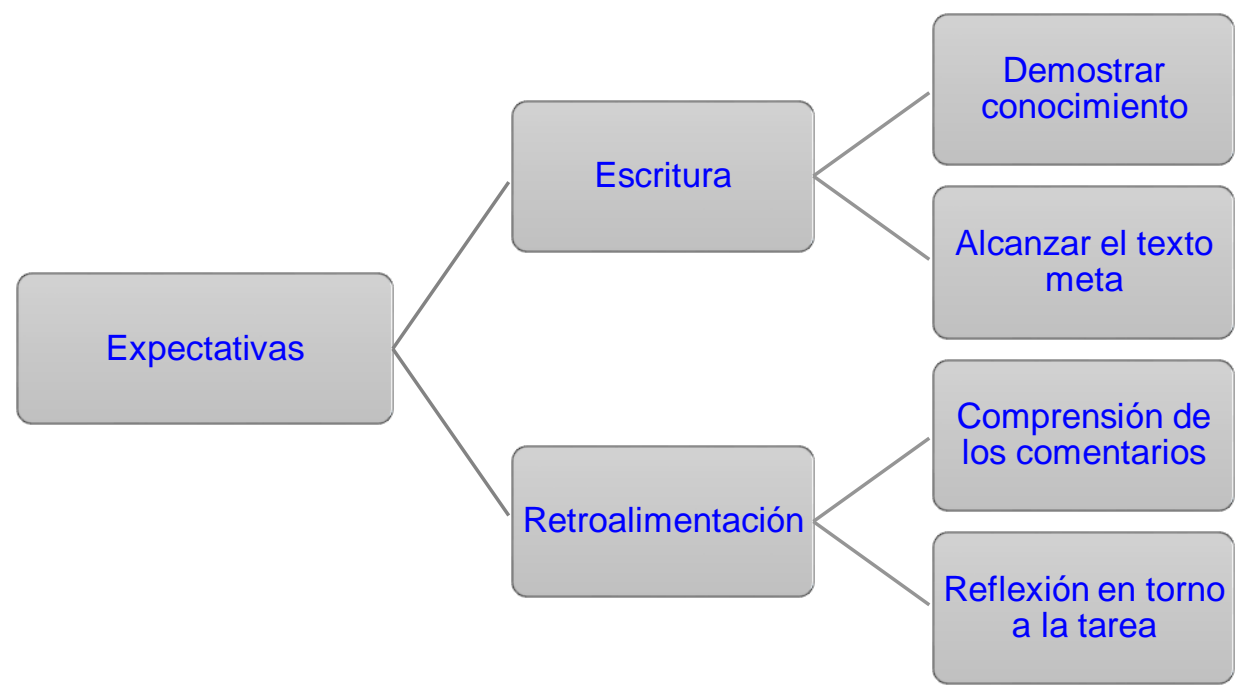

Fig. 1: Expectativas sobre las tareas de escritura y la retroalimentación que proporcionan los docentes.

Respecto a escritura, el análisis sugiere que los docentes de Ingeniería esperan que sus estudiantes demuestren conocimiento sobre la temática de la tarea. Una de las más recurrentes es la elaboración de informes de laboratorio, en los cuales los estudiantes deben hacer comentarios, análisis, reflexiones y sacar conclusiones de los datos obtenidos. La siguiente evidencia obtenida de una entrevista da cuenta de lo anterior: "después viene un parte que es importante para mí que es la de los comentarios, análisis y conclusiones y ahí es donde uno espera que ellos, a partir de la información que obtuvieron de su experimento y de su experiencia, puedan sacar las conclusiones que emanan de las diferentes actividades" (PI 3, comunicación personal, 17 de agosto 2018)

En relación a alcanzar el texto meta, se puede deducir de las entrevistas que en las pautas o rúbricas con que los profesores de Ingeniería evalúan las tareas de escritura, describen las características del texto modelo que esperan que los estudiantes produzcan. El siguiente fragmento es un ejemplo de ello: "Llama mucho la intención eso en el sentido de que hay una pauta, hay una rúbrica digamos, nosotros trabajamos con rúbricas, están establecidas, están las escalas no cierto y las valoraciones, y les decimos, muchachos antes de comenzar cualquier actividad estás son las rúbricas bajo las cuales nosotros vamos a tomar, digamos, conocimiento de los datos que ustedes aportan y de esta manera van a ser calificados por las distintas dimensiones" (PI 1, comunicación personal, 21 de agosto 2018) 
Ahora bien, respecto a las expectativas de los docentes en relación a la retroalimentación que otorgan a sus estudiantes, el análisis identificó los subnodos 'comprensión de los comentarios' y 'reflexión en torno a la tarea'. El primero dice relación con que los docentes esperan que sus estudiantes comprendan los comentarios que les hacen a sus trabajos escritos y el extracto de una entrevista da cuenta de ello "que la debatan, que la cuestionen, que la pregunta que genere conflicto, de que se genere un diálogo..." (PI 4, comunicación personal, 22 de agosto 2018). Finalmente, la segunda subcategoría apunta a la reflexión en torno a la tarea por parte del estudiante. Los profesores de la Facultad de Ingeniería esperan que sus estudiantes mediten o reflexionen respecto a la tarea que están llevando a cabo y que dicha reflexión puede ser gatillada por la retroalimentación que el docente otorga. Esto se evidencia en las palabras de uno de los entrevistados, el cual expresó "Si hacen el trabajo a conciencia y revisan y de verdad meditan de los errores, obviamente es bueno" (PI 6, comunicación personal, 22 de agosto 2018).

La Figura 2 es la representación gráfica de las percepciones que poseen los académicos de Ingeniería respecto a los estudiantes (en relación a la escritura y la retroalimentación) y respecto a la retroalimentación que como docentes proporcionan a los trabajos escritos.

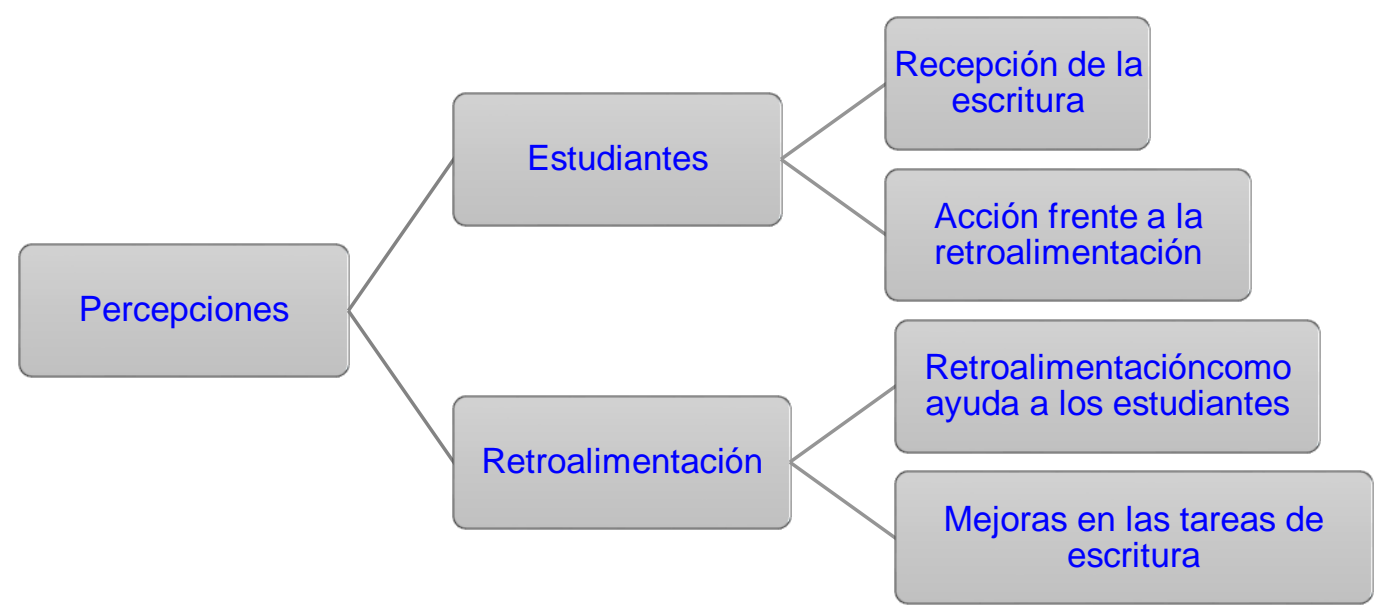

Fig. 2: Percepciones sobre los estudiantes y la retroalimentación proporcionada.

En cuanto a las percepciones de los docentes sobre los estudiantes y la retroalimentación, el análisis muestra que los profesores perciben que sus estudiantes recepcionan las tareas de escritura de mala manera. En torno a esto, uno de los profesores declara "lo perciben como un problema; ya vamos a tener que trabajar de nuevo con un formato, ya nuevamente vamos a tener que gastar energía en tratar de darle palabras a algo que no debiera tenerlo, eso sucede muchas veces" (PI 1, comunicación personal, 21 de agosto 2018).

De igual forma, los profesores declaran que sus estudiantes emprenden acciones a partir de la retroalimentación que reciben en sus trabajos escritos. Al respecto, los profesores entrevistados señalan lo siguiente: "Yo creo que algunos sí se cuestionan y consultan acerca de cómo mejorar, porque no quieren volver a cometer las mismas faltas, porque no son errores digamos muchos son involuntarios, de hecho consideran que pueden mejorar y ellos se ponen las pilas y buscan herramientas, cómo hacerlo, preguntan cómo realmente se hace una vez que los estables están explicados, que uno establece un formato, después tiene que autocompletarlo." (PI 1, comunicación personal, 21 de agosto 2018). "Mira, generalmente lo, digamos la gran mayoría...eeeeh, los aplica y los considera para los posteriores trabajos que realizan ellos." (PI 5, comunicación personal, 23 de agosto 2018).

En cuanto a las percepciones sobre la retroalimentación, y según lo declarado por los profesores, las subcategorías que se desprenden son: a) retroalimentación como ayuda para los estudiantes y b) mejoras en las tareas de escritura. La primera apunta a que la retroalimentación es de utilidad para que los estudiantes puedan corregir sus errores, ver o evidenciar qué hacen y cómo lo están haciendo. Por ejemplo, algunos profesores señalan "la retroalimentación yo creo que es una, una herramienta de evaluación queee ayuda muchísimo a que los alumnos se den cuenta de sus errores y sus virtudes." (PI 5, comunicación personal, 23 de agosto 2018). Sobre las mejoras en las tareas de escritura, los docentes indican "Hay muchachos que de verdad uno ve un crecimiento en el semestre, un desarrollo de sus informes que va de verdad desde, desde un principio que tiene no muy buena calidad a que termina con un informe muy bueno" (PI 6, comunicación personal, 22 de agosto 2018). 


\section{Percepciones y expectativas profesores Facultad de Educación}

Acerca de los docentes de la Facultad de Educación, el análisis permitió construir una representación arbórea respecto a las exceptivas, de las que es posible incorporar subcategorías y subnodos emergentes tal como están organizadas en el discurso.

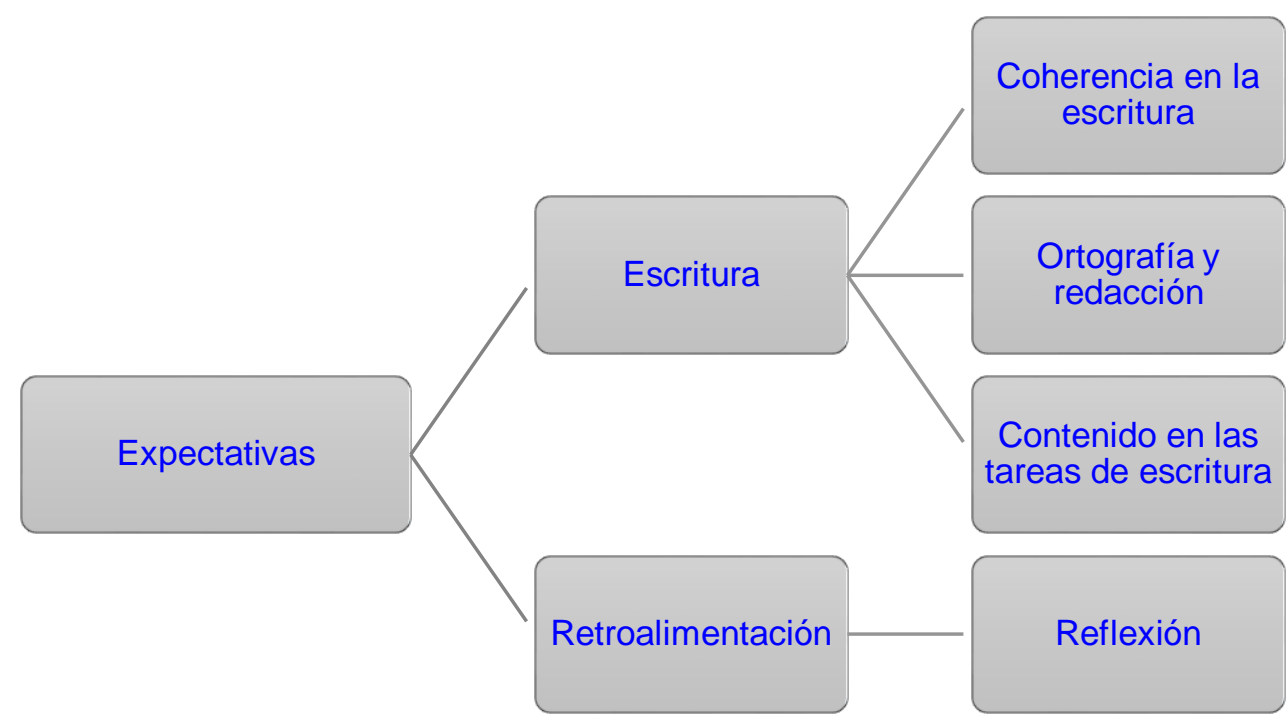

Fig. 3: Expectativas sobre la escritura y la retroalimentación que otorgan a los estudiantes.

En relación a la escritura, es posible señalar que los docentes de la Facultad de Educación esperan que la escritura de los estudiantes demuestre coherencia, buena ortografía y redacción, y que dé cuenta del contenido de la tarea. Cada una de estas características es un subnodo de 'escritura' y respecto a la primera de ellas un docente sostiene que "primero, una redacción coherente, no cierto, en términos de cómo va desarrollando las ideas, los temas, los subtemas, que en cada epígrafe, no cierto, tenga esta bajada no cierto, primero en lo conceptual, el desarrollo de la idea y hacer como una pequeña síntesis de lo que va a trabajando." (PE 4, comunicación personal, 23 de noviembre 2018).

Para el caso de la ortografía y redacción, los entrevistados sostuvieron que los estudiantes deben respetar las normas ortográficas y de redacción. A continuación se dan dos ejemplos de ello "Eeh en el sentido de respetar normas ortográficas, de redacción de otras cosas" (PE 1, comunicación personal, 28 de noviembre 2018) y, además, "y también que haya un buen manejo de lo que es la escritura, es decir, la ortografía, la redacción" (PE 5, comunicación personal, 18 de octubre 2018). Por último, en cuanto al contenido de la tarea, los docentes mencionan que los estudiantes deben seguir las normas de la tarea y que el texto tenga un contenido acorde a lo que se les solicita, un ejemplo de ello es la siguiente referencia "eemmmm que tengan un contenido eeee de acuerdo, a que sigan las normas" (PE 5, comunicación personal, 18 de octubre 2018)

Algunos de los profesores justifican la importancia de corregir elementos como la redacción y la ortografía, debido a que están formando a futuros profesores que serán hablantes/escritores modelo, un ejemplo de esto es el siguiente extracto: "Es muy difícil para uno controlarlo porque nooo la dinámica del tiempo de la universidad no te permite entregarles, lamentablemente, más atención a ese tipos de cosas que son muy importantes, pensando más aún que son futuros profesores." (PE 1, comunicación personal, 28 de noviembre 2018).

Para el caso de las expectativas relacionadas con la retroalimentación, los docentes esperan que sus estudiantes reflexionen en torno al comentario que les realizó. Así, por ejemplo, los académicos declararon que "yo espero que el alumno...eeh sea capaz de darse cuenta de que es lo que, que es lo que le está dificultando el no entender o o o donde está fallando su comprensión de la pregunta a partir de lo que está escribiendo" (PE 2, comunicación personal, 3 de octubre 2018) y "eeemmm, bueno, una que, que lo, que, que reestablezcan y, especialmente, viendo también como tiene, relacionado con la pregunta anterior, como tienen que aprender de los errores, entonces eso también para que quede instaurado en ellos" (PE 3, comunicación personal, 18 de octubre 2018). En cuanto a las percepciones de los profesores de la Facultad de Educación, el análisis de las subcategorías 'percepción de los estudiantes' y 'percepción de la retroalimentación', generó dos subnodos para cada uno de ellos. En la Figura 4 se muestran las percepciones que poseen los profesores de Educación. 
Por un lado, de lo declarado por los docentes en relación a las acciones de los estudiantes frente a la retroalimentación, su visión es que algunos de los estudiantes consideran los comentarios a sus trabajos escritos, pero otros no consideran los comentarios que realiza el profesor. Respecto a ello, uno de los profesores declara "Hay algunos que los consideran, otros que no los consideran ee otro que se preocupan más de mejorar o cuando hacen el próximo trabajo escrito se nota que mejoraron y consideraron los primeros aportes que uno les pudo hacer eemm pero así como hay otros que tampoco lo consideran mucho o mejoran un par de cosas y luego siguen haciéndolo de la misma forma, por lo tanto es relativo" (PE 6 comunicación personal, 20 de diciembre 2018).

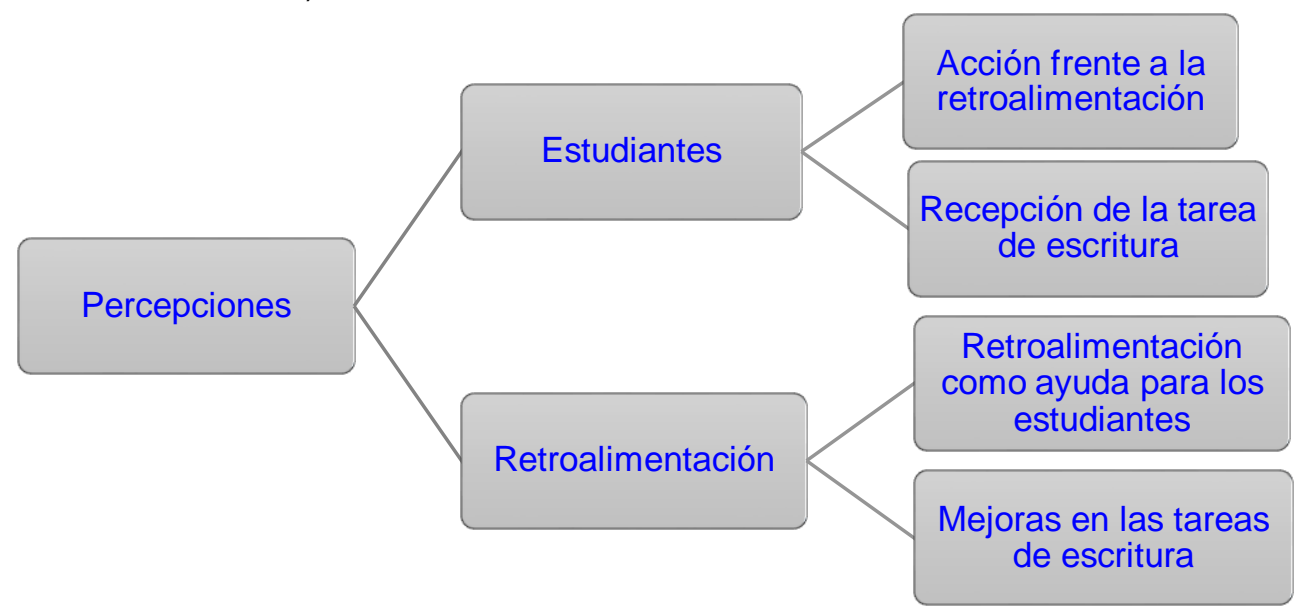

Fig 4: Percepciones sobre los estudiantes y la retroalimentación que los docentes dan a las tareas de escritura.

Por otro lado, en relación con la 'recepción de la tarea', los docentes entrevistados manifiestan que, en general, no hay una buena recepción de las tareas de escritura. Algunos profesores perciben que los estudiantes las consideran como una instancia para aprender, aunque algunos las enfrentan de manera temerosa e insegura en los primeros años de universidad. A continuación, se presentan extractos de entrevistas que dan cuentan de lo dicho anteriormente "ellos lo ven, principalmente, como una instancia para aprender, más que solamente para poder elaborar un texto y tener una nota X ..."(PE 3, comunicación personal, 18 de octubre 2018) y "Eeh, en los primeros dos años de formación son bastante temerosos en temas de escritura y súper inseguros, porque vienen con otro tipo de formación desde los colegios y liceos de donde provienen, por lo tanto cuesta más el proceso de mejorar la escritura" (PE 6 comunicación personal, 20 de diciembre 2018).

Para el caso de las percepciones sobre la retroalimentación, los docentes plantean que la retroalimentación es una herramienta que ayuda a los estudiantes a aprender. Uno de los docentes entrevistados comenta "es un proceso esencial para que el alumno se dé cuenta y haga la metacognición de su aprendizaje, ahí el alumno se da cuenta realmente si aprendió o no aprendió y sobre todo en el tema de la escritura y que lo ven reflejado al final en sus trabajos de tesis sobre todo, que es donde más uno a veces se da cuenta de los errores de los alumnos" (PE 6, comunicación personal, 20 de diciembre 2018). De igual forma, la retroalimentación permite que los estudiantes mejoren en las tareas de escritura. Esto se hace patente en el siguiente aspecto "Yo diría que sí, por todo lo que hemos hablado, porque en general mejora la, en los casos que son así bien bien concretos, la manera de relacionar las ideas en el texto escrito, mejora eso, la complejidad, complejidad de la respuesta que es de manera escrita" (PE 2, comunicación personal, 3 de octubre 2018). Para una mejor comprensión de los resultados obtenidos, al comparar entre los docentes de ambas facultades, se presenta la Tabla 3 , en la que se proporciona un ejemplo por cada una de las subcategorías y por las dos facultades.

\section{DISCUSION FINAL}

A partir de los resultados, es posible plantear que los docentes de Ingeniería esperan que los estudiantes puedan comprender los comentarios, cuestionen aquello que se observa en sus tareas de escritura y mejoren. Además, tanto los docentes de Ingeniería como los de Educación, esperan que la retroalimentación les permita a sus estudiantes reflexionar en torno a la tarea, es decir, meditar, revisar y tomar conciencia en cuanto a los errores y aciertos que tuvieron en la tarea que llevaron a cabo. En cuanto a las percepciones de los participantes respecto al uso que los estudiantes hacen de la retroalimentación a su escritura, es que algunos de ellos la utilizan para mejorar sus trabajos y en evaluaciones posteriores, ya sean certámenes, informes o en otras asignaturas. Sin embargo, los docentes también reconocen que hay otro grupo de estudiantes que no considera los comentarios que reciben en sus trabajos escritos, por lo que las deficiencias en las tareas de escritura persisten en el tiempo. 
Tabla 3. Subcategorías y ejemplos por disciplina.

\begin{tabular}{|l|l|l|}
\hline \multicolumn{1}{|c|}{ Subcategorías } & \multicolumn{2}{|c|}{ Facultad } \\
\hline Escritura & $\begin{array}{l}\text { un poco ver cómo ellos adquirieron estos } \\
\text { conocimientos, cómo los sintetizan interiormente } \\
\text { y después cómo ellos pueden descifrar o dar } \\
\text { una respuesta utilizando todos estos múltiples } \\
\text { conceptos que ellos tiene que manejar para } \\
\text { llegar a la solución (PI 6) }\end{array}$ & $\begin{array}{l}\text { Eee, primero que haya una coherencia } \\
\text { dentro del texto y que si se da una } \\
\text { temática haya un orden lógico, con un } \\
\text { inicio, desarrollo y cierre (PE 6) }\end{array}$ \\
\hline Retroalimentación & $\begin{array}{l}\text { Bueno que...cómo espero que esto les ayude } \\
\text { en el sentido de que si ellos...eeh, se dieron } \\
\text { cuenta dee una falencia o que les falta algo, lo, } \\
\text { lo incluyan o quiten lo que está malo o sigan } \\
\text { manteniendo lo que tienen bueno para que ellos } \\
\text { puedan mejorar su aprendizaje (PI 5) }\end{array}$ & $\begin{array}{l}\text { por ejemplo, en el primer ensayo y que ya } \\
\text { en el segundo curso se nota mejoría, ya, } \\
\text { hay una reflexión, por ejemplo, creo que } \\
\text { uno que se basa en la capacidad reflexiva } \\
\text { que los textos son de mejor calidad en ese } \\
\text { sentido (PE 5) }\end{array}$ \\
\hline $\begin{array}{l}\text { Percepción sobre el } \\
\text { estudiante }\end{array}$ & $\begin{array}{l}\text { si el...la instrucción fue que no se explicaba bien } \\
\text { el orden o no se explicaban bien los datos, la } \\
\text { organización, ellos cambian, o sea, hacen } \\
\text { modificación de conducta en cuanto cuando da } \\
\text { una retroalimentación (PI 4) }\end{array}$ & $\begin{array}{l}\text { hay faltas recurrentes en la medida que } \\
\text { haya una corrección de esa falta yaaa se } \\
\text { elimine, se eliminen (PE 5) }\end{array}$ \\
\hline
\end{tabular}

En ambas disciplinas, lo declarado por los profesores acerca de sus propias prácticas de retroalimentación a la escritura, indica que perciben que la retroalimentación que proveen ayuda a los estudiantes a mejorar su desempeño como escritores. Los docentes perciben que la retroalimentación permite comprender cuál es el objetivo que se persigue con el desarrollo de la tarea; de igual forma, les posibilita a los estudiantes ser conscientes de sus errores y fortalezas, por lo que les permite que lleven a cabo un proceso metacognitivo de su aprendizaje y trabajo llevado a cabo. El análisis de las entrevistas de los participantes están en concordancia con lo planteado por Hounsell et al., (2008), quien postula que la retroalimentación también cumple la función de feedfordward, puesto que permite que el escritor vaya progresando en la tarea de escritura hasta lograr el texto deseado. Para el caso de este estudio, esto se evidencia cuando los docentes hacen mención a los requerimientos de la tarea y lo que se espera que los estudiantes realicen para lograr el objetivo.

Además, tanto los docentes de Ingeniería como los de Educación dan a conocer y entender la relevancia de la retroalimentación en el proceso de aprendizaje de los estudiantes. Cada uno de los profesores expresa la importancia de la retroalimentación en la medida en que esta permite que el estudiante mejore, logre aprender, corrija sus errores y, en lo posible, transferir lo aprendido para un mejor desempeño en las siguientes tareas de escritura que les asignen.

El análisis de las expectativas de los participantes revela diferencias respecto a lo que se espera de las tareas de escritura de los estudiantes en ambas disciplinas. Las expectativas en torno a las tareas de escritura en Ingeniería y Educación apuntan a factores diversos. Lo declarado por los docentes de Ingeniería indica que sus expectativas se refieren a que los estudiantes den cuenta de los conocimientos que adquirieron con el análisis de la información recabada para realizar la tarea. Estos docentes también esperan que sus estudiantes alcancen el texto meta que se les describe en las instrucciones y rúbricas o pautas de evaluación con las cuales se les calificará, lo que da cuenta de una función epistémica de la escritura.

Desde el punto de vista de los académicos de la Facultad de Educación, la escritura debe dar cuenta de una buena redacción, ortografía, coherencia y contenido en lo que se escribe, dado que se está formando a formadores que serán un modelo de escritura para sus estudiantes. Es decir, los profesores esperan que los estudiantes de Educación, a través de los textos que escriben, enseñen a otros a escribir. En síntesis esta investigación ha permitido develar la enseñanza de la escritura a través del currículum, el cual es un enfoque sobre la enseñanza de la lectura y la escritura de forma situada, disciplinarmente especializada y pedagógicamente centrada en las formas de escritura de los distintos espacios académicos y profesionales (Bazerman et al., 2016) y, además, evidenciar que la retroalimentación en la escritura dentro de cada materia es necesaria, tanto por su potencial epistémico, como por tratarse de usos del lenguaje con especificidades disciplinares, que no se aprenden sino situadamente (Artemeva, 2008).

\section{CONCLUSIONES}

De acuerdo al trabajo presentado, a los resultados obtenidos, y a la discusión realizada, se pueden plantear las siguientes conclusiones principales:

1.- Los docentes de Ingeniería esperan que los estudiantes puedan comprender los comentarios, cuestionen aquello que se observa en sus tareas de escritura y mejorar. 
2.- Los docentes de ambas facultades esperan que esta retroalimentación les permita reflexionar en torno a la tarea; es decir, meditar, revisar y tomar conciencia en cuanto a los errores y aciertos que tuvieron en la tarea que llevaron a cabo.

3.- Los docentes declaran que algunos de los estudiantes actúan frente a la retroalimentación, por ende, mejoran en sus trabajos y las evaluaciones posteriores, certámenes, informes o en otras asignaturas.

4.- Los docentes reconocen que hay otro grupo de estudiantes que no considera los comentarios que reciben en sus trabajos escritos, por lo que las deficiencias en los trabajos persisten en el tiempo.

5.- Los docentes de ambas facultades declaran que la retroalimentación ayuda a los estudiantes a mejorar sus tareas de escritura.

6.- La retroalimentación permite comprender cuál es el objetivo que se persigue con el desarrollo de la tarea y les posibilita ser conscientes de sus errores y fortalezas, permitiendo que realicen un proceso metacognitivo de su aprendizaje.

\section{AGRADECIMIENTOS}

Al equipo del proyecto Fondecyt 1180586, en el cual se enmarca esta investigación, por su colaboración, críticas y observaciones realizadas durante el desarrollo de la investigación.

\section{REFERENCIAS}

Alvira, R. The impact of oral and written feedback on EFL writers with the use of screencasts, DOI: 10.15446/profile.v18n2.53397, Profile Issues in TeachersProfessional Development, 18(2), 79-92 (2016).

Arancibia Gutiérrez, B., Tapia-Ladino, M., y Correa Pérez, R. La retroalimentación durante el proceso de escritura de la tesis en carreras de pedagogía: Descripción de los comentarios escritos de los profesores guías. DOI:10.4067/S071809342019000200242, Revista Signos, 52(100), 242-264 (2019).

Artemeva, N. Toward a unified social theory of genre learning. DOI: 10.1177/1050651907311925, Journal of Business and Technical Communication, 22(2), 160-185 (2008).

Bautista, C. Proceso de la investigación cualitativa: Epistemología, metodología y aplicaciones, Colombia: Manual Moderno. (2011).

Bazerman, C., Little, J., y otros cuatros autores más. Escribir a través del Currículum. Una guía de referencia (2016).

Boud, D. and Molloy, E. El feedback en educación superior y profesional: comprenderlo y hacerlo bien, Narcea Ediciones (2015).

Brown, S. y Pickford, R. Evaluación de habilidades y competencias en educación superior, Madrid, España: Narcea Ediciones (2014).

Camps, A. Castelló, M. La escritura académica en la Universidad. ISSN: 1887-4592, Revista de Docencia Universitaria, 11(1), 17-36 (2013).

Canabal, C., y Margalef, L. La retroalimentación: la clave para una evaluación orientada al aprendizaje, Profesorado, Revista de Currículum y Formación del Profesorado, 21(2), 149-170 (2017).

Carlino, P., Escribir, leer, y aprender en la universidad. Una introducción a la alfabetización académica. Fondo de Cultura Económica de Argentina, 4-9 (2005).

Carlino, P., Didáctica de la lectura en la universidad. Ámbito de Encuentros, 2(1), 47-67 (2008).

Contreras-Pérez, G., y Zúñiga-González, C. G., Concepciones de profesores sobre retroalimentación: una revisión de la literatura, Magis, Revista Internacional de Investigación en Educación, 9(19), 69-90 (2017).

Côrte, M. I., La escritura académica: En la formación universitaria, Narcea: Madrid (2018).

Chan, P. E., Konrad, M., Gonzalez, V., Peters, M. T., y Ressa, V. A. The critical role of feedback in formative instructional practices, DOI: 10.1177/1053451214536044, Intervention in School and Clinic, 5O(2), 96-104 (2014).

Chappuis, J., Stiggins, R., Chappuis, S. y Arter, J. Classroom assessment for student learning: Doing it right, using it well. Assessment Training Institute (2012).

Christiansen, M. S. y Bloch, J. Papers are never finished, just abandoned': The role of written teacher comments in the revision process. Journal of Response to Writing, 2(1), 6-42 (2016).

Dowden, T., Pittaway, Sh., Yost, H. y McCarthy, R. Students' perceptions of written feedback in teacher education: Ideally feedback is a continuing two-way communication that encourages progress. DOI: 10.1080/02602938.2011.632676, Assessment \& Evaluation in Higher Education, 38(3), 349-362 (2013).

Duijnhouwer, H. Feedback effects on student's writing motivation, process and performance. Tesis doctoral, Universiteit Utrecht, Utrecht, Países Bajos (2010).

Ellis, R. A typology of written corrective feedback types. DOI: 10.1093/elt/ccn023, ELT Journal, 63(2), 97-107 (2009). 
Ferris, D. Second language writing research and written corrective feedback in SLA Intersections and Practical Applications, DOI: 10.1017/S0272263109990490, Studies in Second Language Acquisition, 32, 181-201 (2010).

Hartshorn, K. y Evans, N. The Effects of Dynamic Written Corrective Feedback: A 30Week Study. Journal of Response to Writing, 1(2): 6-34 (2015).

Hounsell, D., McCune, V., Hounsell, J., y Litjens, J. The quality of guidance and feedback to students. DOI: 10.1080/07294360701658765, Higher Education Research \& Development, 27(1), 55-67 (2008).

Huisman, B., Saab, N., van Driel, J y van den Broek, P. Peer feedback on academic writing: undergraduate students' peer feedback role, peer feedback perceptions and essay performance. DOI: 10.1080/02602938.2018. Assessment \& Evaluation in Higher Education, 43(6), 955-968. (2018).

Hyland, K. Genre, discipline and identity. Journal of english for academic purposes 19, $32-43$ (2015).

Irigoyen, J., Carpio, C., y otros cuatro autores. Efecto de los diferentes tipos funcionales de retroalimentación y su presentación parcial en el entrenamiento y transferencia de desempeños efectivos. Revista sonorense de psicología, 16(1), 23-31 (2002).

Mares, A., Martínez, R., y Rojo, H. Concepto y expectativas del docente respecto de sus alumnos considerados con necesidades educativas especiales, Revista mexicana de investigación educativa, 14(42), p.p. $969-996$ (2009).

Marinkovich, J., y Córdova, A. La escritura en la universidad: Objeto de estudio, método y discursos, http://dx.doi.org/10.4067/S0718-09342014000100003, Revista Signos, 47(84), 40-63 (2014).

Marinkovich, J., Sologuren, E. y Shawky, M. The process of academic literacy in Civil Engineering Computer Science. An approach to academic writing and its genres in a learning community. DOI: 10.5209/CLAC.60520, Círculo de Lingüística Aplicada a la Comunicación, (2018).

Martínez, F. G. L., y Vargas, L. A. T. Retroalimentación formativa para estudiantes de educación a distancia, RIED. Revista Iberoamericana de Educación a Distancia, 17(2), 197-221 (2014).

Monedero, J. Bases teóricas de la evaluación educativa. Málaga- España: Ediciones Aljibe (1998).

Navarro, F., Uribe Gajardo, F., Lovera Falcón, P., y Sologuren, E. Encuentros con la escritura en el ingreso a la educación superior: representaciones sociales de estudiantes en seis áreas de conocimiento, Ibérica, 38, 75-98 (2019).

Ochoa-Sierra, L., y Moreno-Mosquera, E. Analysis of Written Comments from Graduate Thesis Advisors, DOI: 10.17227/rce.num76-5725, Revista Colombiana de Educación, (76), 143-171 (2019).

Ortiz, G. Precisión de descripciones, retroalimentación y conocimiento de la finalidad de la descripción poscontacto sobre la ejecución, elaboración y transmisión de descripciones, Acta Comportamentalia, 18 (2), 189-213 (2010).

Padilla, C., y López, E. Prácticas de retroalimentación en aulas universitarias de humanidades: Comentarios digitales docentes y perfiles estudiantiles de escritor, DOI: 10.4067/S0718-09342019000200330, Revista Signos. Estudios de Lingüística, 52(100) (2019).

Rosales, C. Criterios para una evaluación formativa, ISBN 10: 8427704763 / ISBN 13: 9788427704763 , Narcea Ediciones (2014).

Tapia-Ladino, M., Pérez, R. C., y Gutiérrez, B. A. Retroalimentación con Comentarios Escritos de Ajuste al Género (CEAG) en el proceso de elaboración de tesis de Programas de Formación de profesores. ISSN 0716-0542, Lenguas Modernas, (50), 175-192 (2017).

Vargas, L. Sobre el concepto de percepción, ISSN: 0188-7017, Alteridades, 4(8) (1994).

Valle, A. y Núñez, J. C., Las expectativas del profesor y su incidencia en el contexto institucional, Revista de educación, (290), 293-319 (1989).

Wisker, G., Robinson, G., Trafford, V., Warnes, M. y Creighton, E. From supervisory dialogues to successful PhDs: Strategies supporting and enabling the learning conversations of staff and students at postgraduate level, DOI: 10.1080/13562510309400, Teaching in Higher Education, 8(3), 383-397 (2003).

Yallop, R.M.A., Leijen, D.A.J. The perceived effectiveness of written peer feedback comments within 12 English academic writing courses. DOI: 10.5128/ERYa14.15, Eesti Rakenduslingvistika Uhingu Aastaraamat, 14, pp. 247-271 (2018) 
